\title{
Group IIC intron mobility into attC sites involves a bulged DNA stem-loop motif
}

\author{
GRÉGORY LÉON ${ }^{1,2}$ and PAUL H. ROY ${ }^{1,2}$ \\ ${ }^{1}$ Centre de Recherche en Infectiologie, Centre Hospitalier Universitaire de Québec, Université Laval, Québec G1V 4G2, Canada \\ ${ }^{2}$ Département de Biochimie et de Microbiologie, Faculté des Sciences et de Génie, Université Laval, Québec G1V 4G2, Canada
}

\begin{abstract}
Bacterial group IIC introns are a subclass of group II intron ribozymes that are typically located downstream from transcriptional terminators. Class IIC-attC introns constitute a monophyletic subset of subgroup IIC, which preferentially insert into site-specific recombination sequences for integron integrases (attC). attCs are a diverse family of nucleotide sequences composed of conserved inverted repeats that flank a variable, but palindromic, central region. In this study, we used both PCR and colony patch hybridization methods to determine the basis for recognition of the attC $C_{\text {aadA1 }}$ stem-loop motif by the Serratia marcescens intron (S.ma.I2) in vivo. The quantitative results showed that mobility into the wild-type site occurs at a frequency of $18 \%$, and is strongly biased by the orientation of the homing site relative to the direction of DNA replication. S.ma.I2 mobility results into mutant attC $_{\text {aadA1 }}$ sites are consistent with recognition of stem-loop motifs in unwound DNA. The homing frequency results showed that, while the entire attC sequence is not necessary for recognition of the insertion site, short deletions of the attC stem-loop motif inhibited the intron mobility. Moreover, our data show that $S$.ma.I2 requires a bulged base in the folded attC stem for high homing frequency. We demonstrate that the IBS1/IBS3 motifs and two bulge bases conserved among attCs determine S.ma.I2 homing specificity for the attC bottom strand. These results suggest that class IIC-attC introns tolerate attC variation by recognition of a bulged hairpin DNA motif rather than a specific sequence.
\end{abstract}

Keywords: cassette; integron; resistance; retrohoming; ribozyme

\section{INTRODUCTION}

Group II introns are retroelements coding for a large structured catalytic RNA and a multidomain intron-encoded protein (IEP), which has maturase, reverse transcriptase (RT) and in some cases endonuclease activities (Lambowitz and Zimmerly 2004). They are found in organelles (from lower eukaryotes and higher plants), and in bacterial and archaeal genomes (Ferat and Michel 1993; Martinez-Abarca and Toro 2000; Bonen and Vogel 2001; Toro 2003; Pyle and Lambowitz 2006). Group II introns share very little sequence similarity but have a conserved intron RNA secondary structure, composed of six structural domains, which coevolved with the intron's RT (Toor et al. 2001). Phylogenetic analyses, based on IEP sequences, allow separation of group II introns into several lineages (chloroplast-like, mitochondrial-like, bacterial classes A-F) (Zimmerly et al. 2001;

Reprint requests to: Paul H. Roy, Centre de Recherche en Infectiologie, Centre Hospitalier Universitaire de Québec, Université Laval, Pavillon CHUL, Local RC-709, 2705 Boulevard Laurier, Québec G1V 4G2, Canada; e-mail: paul.roy@crchul.ulaval.ca; fax: (418) 654-2715.

Article published online ahead of print. Article and publication date are at http://www.rnajournal.org/cgi/doi/10.1261/rna.1649309.
Simon et al. 2008). Together, the IEP and the spliced intron RNA form a ribonucleoprotein (RNP) complex, which mediates intron mobility and invasion of DNA targets (Zimmerly et al. 1995; Matsuura et al. 1997; Cousineau et al. 1998). The intron RNA catalyzes excision (splicing) from RNA transcripts and integration (reverse splicing) into one strand of the new allele target. The IEP facilitates these reactions by stabilizing the intron RNA structure (maturase activity) and by cleaving the strand opposite to the reverse spliced intron (endonuclease activity) to generate the primer for reverse transcription of the intron RNA (Lambowitz and Zimmerly 2004). However, over half of the IEPs in bacteria lack the endonuclease domain (Toro 2003). It has been shown that nascent strands at DNA replication forks can also serve as primers for reverse transcription of the intron RNA (by the IEP's RT activity) in the absence of second strand cleavage (Martinez-Abarca et al. 2000; Jimenez-Zurdo et al. 2003; Munoz-Adelantado et al. 2003; Zhong and Lambowitz 2003). This mechanism of mobility shows a pronounced strand bias and dependence on replication. Homing site recognition uses both the IEP and base pairing of the intron RNA with a specific DNA sequence (Guo et al. 1997). In a first step, the RNP binds DNA nonspecifically and then 
searches for a small number of specific bases in the distal $5^{\prime}$-exon and $3^{\prime}$-exon regions of the target site via major groove interactions. These base interactions enhance local DNA unwinding and a conformational change associated with identification of the specific binding site (Singh and Lambowitz 2001). In a second step, two exon binding sites (EBS1 and EBS2), located in the intron RNA, are involved in $5^{\prime}$ exon recognition through base pairing interactions with the target's intron binding sites (IBS1 and IBS2). Similarly, the $3^{\prime}$ exon is recognized by either the $\delta-\delta^{\prime}$ or EBS3IBS3 interactions (Jacquier and Michel 1987; Michel and Ferat 1995).

Bacterial class IIC introns are a phylogenetic subclass of group II introns that exhibit unique RNA structure (e.g., the EBS2 motif is missing; they have shorter EBS1-EBS3 motifs and a shorter catalytic domain 5) (Toor et al. 2001; Toor et al. 2008) and self-splicing properties (e.g., they generally self-splice in vitro through a hydrolytic pathway resulting in linear intron intermediates) (Podar et al. 1998). Furthermore, it was shown that a stem-loop motif located a few nucleotides upstream of the IBS1 was implicated in recognition of the $5^{\prime}$ exon during both splicing and reversesplicing events (Toor et al. 2006; Robart et al. 2007). The targeted stem-loop structures are mostly transcriptional terminators (Granlund et al. 2001), which show low levels of sequence identity but have conserved stem-loop structures. Interestingly, a specialized lineage within class $\mathrm{C}$ introns, named class IIC-attC introns, preferentially inserts into the inverted repeats of integron attC sites (Centron and Roy 2002; Quiroga et al. 2008).

attC sites (or 59-base elements) are site-specific recombination sequences located at the $3^{\prime}$ end of integron gene cassettes (Hall et al. 1991). Integron integrases mediate sitespecific recombination of DNA sequences flanking open reading frames or structural genes (e.g., drug resistance genes) using two distinct sites, a proximal attI and a cassetteassociated $a t t C$ site. The key elements of integrons are: an integrase gene (intI), a promoter for expression of cassettes $\left(\mathrm{P}_{\mathrm{c}}\right)$, and a recombination site (attI) (Fig. 1A; Stokes and Hall 1989; Mazel 2006). Alignment of different attCs shows that they vary in length from 57 to 141 base pairs (bp), and that their sequence similarities are primarily restricted to the boundaries, which correspond to two inverted integrasebinding repeats, $1 \mathrm{~L}-2 \mathrm{~L}$ separated by a spacer of $5 \mathrm{bp}$ and $2 \mathrm{R}$ 1R separated by 6 bp (Fig. 1B; Francia et al. 1997; Stokes et al. 1997). The central part between the $2 \mathrm{~L}$ and $2 \mathrm{R}$ repeats varies both in length and sequence but is an imperfect inverted repeat. It was shown by electrophoretic mobility shift assays (EMSA) that integron integrase preferentially binds to single-strand DNA of the attCs (Francia et al. 1999). Interestingly, binding of integrase is strand specific for the bottom strand (bs) of the attC. It was suggested that formation of a cruciform (i.e., stem-loop structure on each strand) and imperfections in the inverted repeats (Fig. 1B) were of importance for $a t t C$ site recognition by integrase and for further recombination reactions (Hall et al. 1991; Stokes et al. 1997; Francia et al. 1999). A recent study demonstrates that integrase specificity for the attC bottom strand is due to the presence of two conserved asymmetrical bases ( $T$ and G) that bulge out from the folded DNA sequence (Fig. 1C; Johansson et al. 2004). The crystal structure of the VchIntIA-VCR $\mathrm{V}_{\mathrm{bs}}$ synapse (class 4 integron integrase monomers bound to two antiparallel $\mathrm{VCR}_{\mathrm{bs}}$ duplexes) showed that integrase binds its attC substrate as a dimer (MacDonald et al. 2006). The extrahelical base $\mathrm{T}$ is bound by cisinteraction with one integrase subunit, and is important for DNA site recognition, whereas the extrahelical base $\mathrm{G}$ is bound by trans-interaction with a second integrase subunit bound to the other $\mathrm{VCR}_{\mathrm{bs}}$. The trans-interactions are important features to hold the synaptic complex together during recombination.

To address how bacterial class IIC-attC introns insert specifically into the bottom strand of attC sites, we used the S.ma.I2 intron in a two-plasmid mobility assay. S.ma.I2 was found in a class 1 integron, from the multiresistant clinical isolate Serratia marcescens SCH909, adjacent to the aadB (aminoglycoside resistance) gene cassette (Centron and Roy 2002). We generated several mutants of the att $C_{a a d A 1}$ site from the aadA1-qacE cassette junction in Tn21 (Fig. 1B) in order to find stem-loop features that influence intron homing. We used this site because it is widespread among plasmid-borne cassettes and is almost a perfect palindromic sequence (Fig. 1C), but most importantly because of previous observations in vitro demonstrating specific binding of integrase to the bottom strand of att $C_{\text {aadAl }}$ (Francia et al. 1999; Johansson et al. 2004; Bouvier et al. 2005). The quantitative results showed that mobility into the wild-type (WT) site occurs at a frequency (the fraction of homing site plasmids containing introns) of $18 \%$ and is strongly biased by the orientation of the homing site relative to the direction of DNA replication. S.ma.I2 mobility results into mutant att $C_{\text {aadA } 1}$ sites are consistent with recognition of stem-loop motifs in unwound DNA, as in the Bacillus halodurans class IIC intron (Robart et al. 2007). The homing frequency results showed that, while the entire $a t t C$ sequence is not necessary for recognition of the insertion site, short deletions of the attC stem-loop motif inhibited the intron mobility. Moreover, our data show that S.ma.I2 requires a bulged base in the folded attC stem for high homing frequency. We demonstrate that the IBS1/IBS3 motifs and two bulge bases conserved among attC sites are determinants for S.ma.I2 homing specificity for the attC bottom strand.

\section{RESULTS}

\section{In vivo assay of mobility into the wild-type attC $_{\text {aadA1 } 1}$ site}

To address how bacterial class IIC-attC introns insert specifically into the bottom strand of attC sites, we used 
A
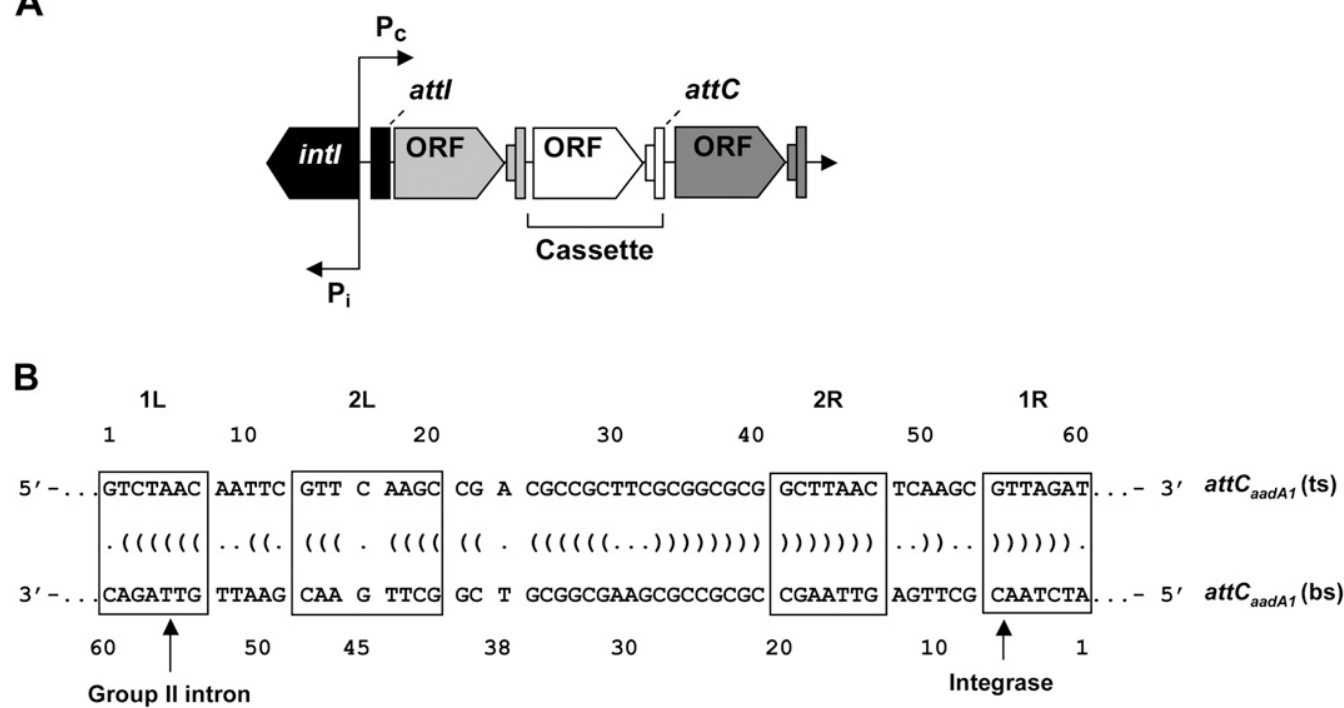

C $\operatorname{attC}_{\text {aadA1 }}(\mathrm{ts})$

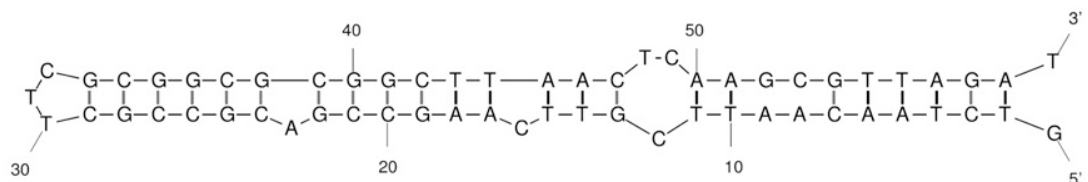
10

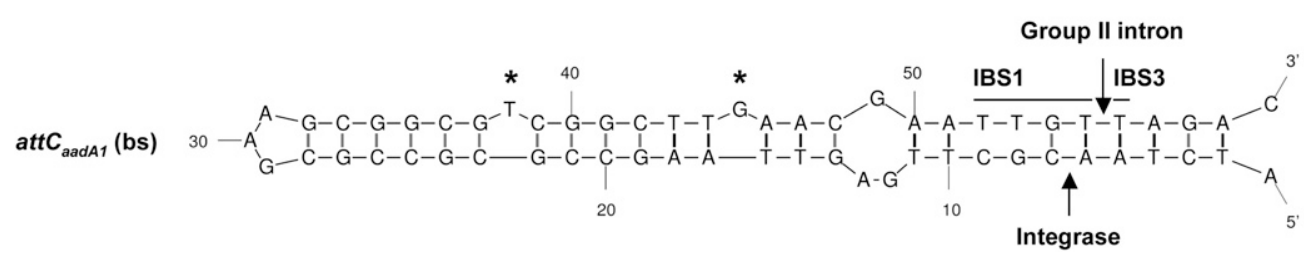

FIGURE 1. Integron structure and $a t t C$ recombination sites. $(A)$ General schematic representation of an integron. intI, integrase gene; $\mathrm{P}_{\mathrm{i}}$, integrase promoter; $\mathrm{P}_{c}$, cassette promoter; attI, integrase recombination site (integrase specific); ORF, cassette open reading frame; attC, integrase recombination site (cassette specific). (B) Sequence of the att $C_{\text {aadAl }}$ site. $2 \mathrm{~L}-2 \mathrm{R}$ and $1 \mathrm{~L}-1 \mathrm{R}$ boxes are inverted repeats; arrows, integrase recombination site and intron insertion site; Vienna output (dots and parentheses between the top-strand [ts] and bottom-strand [bs] sequences), base pairings from a potential single-stranded secondary structure based on MFOLD prediction. $(C)$ Predicted stem-loop structures of the attC top- and bottom-strand sequences based on MFOLD predictions. IBS1 and IBS3, intron binding sites 1 and 3; asterisk $\left({ }^{*}\right)$, extrahelical bases T38 and G45 upon folding of the attC bottom strand.

an Escherichia coli two-plasmid mobility assay in which S.ma.I2 inserted into WT or mutant att $C_{a a d A 1}$ sites (Figs. 2, 3 ). For each target, oligonucleotides of both complementary DNA strands were annealed, cloned, and tested as homing sites (Supplemental Table 1). Intron mobility was screened using a qualitative PCR method in combination with a quantitative colony patch hybridization method to measure and compare intron mobility frequencies into WT and mutant att $C_{a a d A 1}$ sites (Table 1; see Materials and Methods). Three targets were cloned in both orientations such that reverse transcription of the inserted intron RNA could potentially use either a nascent lagging (LAG) or leading (LEAD) DNA strand as a primer at the replication fork. These clones were tested in order to detect a probable strand bias for homing in our experiment. It was previously reported that different group II introns with IEPs lacking the endonuclease domain prefer either a nascent lagging strand (Martinez-Abarca et al. 2004) or leading strand (Zhong and Lambowitz 2003) as a primer. Figure 4 shows the orientation of intron insertion using a highly sensitive PCR-based approach, whereas Figure 5 shows degrees of intron insertion (semiquantitatively) without information on the orientation. Mobility assays with the WT site show that S.ma.I2 homes specifically into the attC bottom strand (Fig. 4). Sequencing of the PCR amplicon confirmed that S.ma.I2 used the expected 5'-TTGT/T sequence (IBS1/IBS3, respectively) as its intron binding sites (data not shown) (Quiroga et al. 2008). However, comparison of intron mobility levels into the WT (LAG) and WT (LEAD) sites is consistent with a replication orientation bias (Fig. 5). Indeed, quantitative results suggest that reverse transcription of the inserted intron RNA preferred a nascent lagging (LAG) strand DNA as a primer over a leading (LEAD) strand DNA $(18.0 \pm 2.5 \%$ and $<1.0 \%$ 


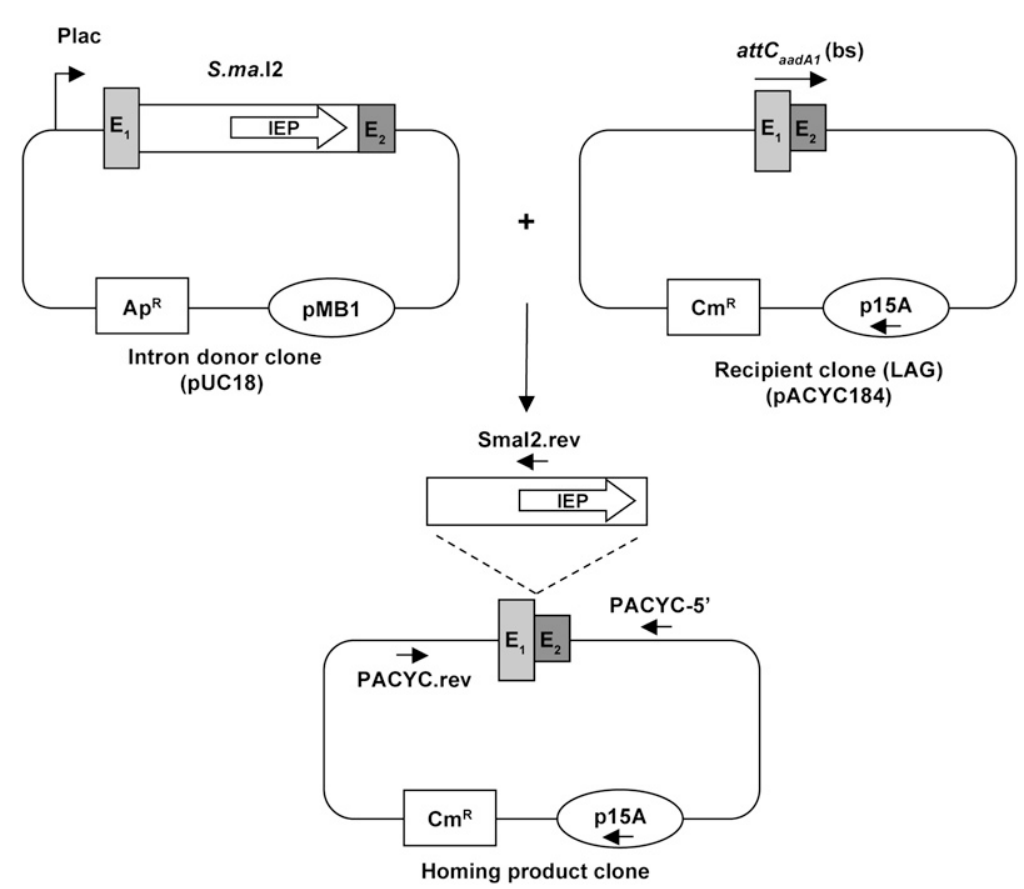

FIGURE 2. Schematic of the two-plasmid mobility assay in E. coli (see the Materials and Methods section for a more detailed protocol). $\mathrm{E}_{1}$ and $\mathrm{E}_{2}$, exon sequences; IEP, S.ma.I2 intron encoded protein; Plac, lac promoter; $\mathrm{Ap}^{\mathrm{R}}$ and $\mathrm{Cm}^{\mathrm{R}}$, ampicillin and chloramphenicol resistance genes; pMB1 and p15A, plasmid replication origins.

homing frequencies, respectively) (Table 1). Figure 4 shows that despite the low homing frequencies observed using the LEAD construct, mobility events into the WT (LEAD) site were amplified by PCR. The identities of the amplicons were confirmed by sequencing (data not shown). In order to find the structural determinants for recognition of the $a t t C_{a a d A 1}$ site, we designed and cloned 26 att $C_{a a d A 1}$ mutants and tested them as potential target sites for S.ma.I2, taking into account the effect of homing site orientation in pACYC184 (Fig. 3).

\section{Mutation of the $5^{\prime}$-exon stem lengths}

The att $C_{\text {aadAl }}$ bottom strand is almost a perfect palindromic sequence except for the "spacer" bases (between the $1 \mathrm{~L}-1 \mathrm{R}$ and $2 \mathrm{~L}-2 \mathrm{R}$ boxes), the two conserved asymmetrical bases (T38 and G45) and the central loop (GAA) (Fig. 1C). According to the predicted secondary structure, we designed seven stem-loop mutants $(\Delta 1-\Delta 7)$, which are $5^{\prime}$-end truncations of the WT att $C_{a a d A 1}$ bottom strand sequence (homing strand) (Fig. 3A). The homing results showed that the minimal target was a 44-bp att $C_{\text {aadAl }}$ (mutant $\Delta 5$ ) that includes $39 \mathrm{bp}$ of $5^{\prime}$ exon (Fig. 4). The $5^{\prime}$ exon sequence can fold into a stem (12 bp) and loop (3 nucleotides [nt]) motif, which is separated from the IBS1 sequence ( $5^{\prime}$-TTGT) by a 7-nt spacer (Fig. 3A). For this target, residual S.ma.I2 mobility was $1.8 \pm 1.0 \%$, whereas $18.0 \pm 2.5 \%$ mobility was observed for the WT attC aadAl (Table 1). Further deletion resulted in the absence of detectable mobility into the homing site (Fig. 4). The homing frequencies showed that, while the entire att $C_{a a d A 1}$ sequence is not necessary for intron homing, even short deletions, like the 7-bp $\Delta 1$ mutant, inhibited the intron mobility (8.0 \pm $0.7 \%)$. Interestingly, base deletions of the attC ranging from the $1 \mathrm{R}$ to the beginning of the $2 \mathrm{R}$ inverted repeats $(\Delta 1-\Delta 4)$ resulted in a $\sim 50 \%$ (mean) diminution of the homing reaction in vivo. Additional deletions, including half $(\Delta 5)$, or all, of the $2 \mathrm{R}$ site ( $\Delta 6$ and $\Delta 7)$, resulted in a $\sim 90 \%$ diminution or a complete inhibition of the in vivo mobility, respectively.

\section{Mutation of the stem pairings}

For this mobility assay, we substituted five nucleotides in att $C_{a a d A 1}$ in order to disrupt the stem before the central loop (Fig. 3B). It was shown that an identical stem mutation on att $C_{a a d A 7}$ inhibits the recombination frequency by integrase (Bouvier et al. 2005). Here, intron mobility into the attC bottom strand was detected by PCR (Fig. 4) but quantitative results showed an important loss of efficiency $(<1.0 \%)$ (Table 1$)$. This result was confirmed by the absence of PCR amplicon using two pACYC184-specific primers that flank the attC site (Fig. 5).

\section{Mutation of IBS1 or IBS3}

Retrohoming of group II introns is normally highly specific because of base pairings between the intron RNA and the DNA exons. Bacterial class IIC intron base pairings are limited to the short IBS1-EBS1 and IBS3-EBS3 interactions (Robart et al. 2007). Here, we designed three IBS1 motif mutants (IBS1-1 to IBS1-3) and one IBS3 mutant (IBS3) (Fig. 3C). PCR results confirmed the importance of a specific IBS1 sequence $\left(5^{\prime}\right.$-TTGT) and, to a lesser proportion, a specific IBS3 (T) base. Indeed, a weak PCR amplicon was obtained only for the first IBS1 mutant (IBS1-1) and a strong PCR amplicon for the unique IBS3 mutant (IBS3) (Fig. 4). Hybridization results confirmed the PCR results obtained with the IBS1-1 and IBS3 mutants, respectively, as the corresponding homing frequencies were $<1.0 \%$ and $4.6 \pm 2.9 \%$ (Table 1 ).

\section{Deletion of the extrahelical bases T38 and G45}

Quantitative results of mobility of S.ma.I2 into att $C_{\text {aadAI }}$ with mutated $5^{\prime}$-exon stems (above) showed that short 
A

\begin{tabular}{|c|c|}
\hline & 10 \\
\hline WT & $.1(1)(1 \ldots)(1 \ldots)(1$ \\
\hline WT & $5^{\prime}-\ldots$ ATCTAACGCTTGAGTT \\
\hline$\Delta 1$ & ... aggatcc. \\
\hline$\Delta 2$ & ...agaggatcc...... \\
\hline$\Delta 3$ & ...gcgtagaggatcc... \\
\hline$\Delta 4$ & ...ggcgtagaggatcc. . \\
\hline$\Delta 5$ & ...cggcgtagaggatccg \\
\hline$\Delta 6$ & ...cgtccggcgtagagga \\
\hline$\Delta 7$ & ...gccacgatgcgtccgg \\
\hline
\end{tabular}

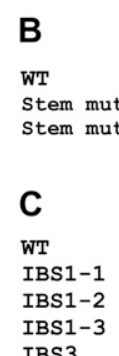

$5^{\prime}-\ldots$ ATCTAACGCTTGAGTT

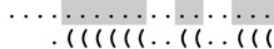

\section{AAGCCG}

i $(1(i)$

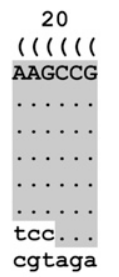

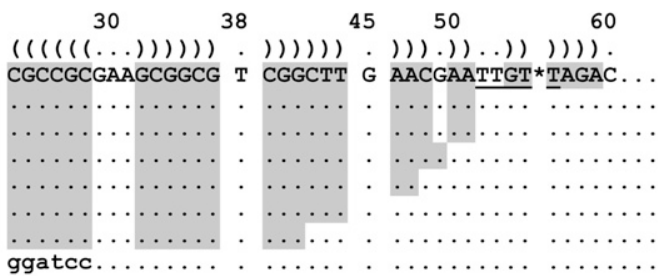

CGCCGCGAAGCGGCG T CGGCTT G AACGAATTGT $*$ TAGAC...

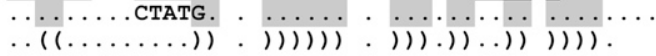

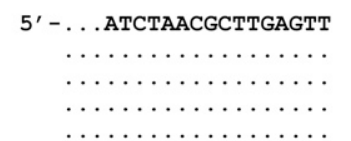

AAGCCG

$\cdots \cdots$

$\ldots \ldots$

$\cdots \cdots$

$\cdots \cdots$

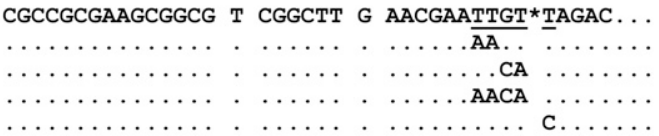

$5^{\prime}-\ldots$ ATCTAACGCTTGAGTT

. W . W .

$\ldots \ldots \ldots \ldots \ldots$

AAGCCG

$\ldots \ldots$

$\ldots \ldots$

$\ldots \ldots$

CGCCGCGAAGCGGCG T CGGCTT G AACGAATTGT ${ }^{*}$ TAGAC...

$\ldots \ldots \ldots \ldots \ldots-\ldots \ldots \ldots \ldots$

$\ldots \ldots \ldots \ldots$ т $\ldots \ldots$ - $\ldots \ldots \ldots$

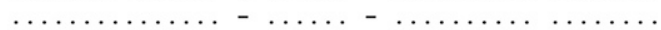

E

WT

T38A

T38G

G45A

G45C

G45T

G45GG

T38A+G45C

T38G+G45T

$5^{\prime}-\ldots$ ATCTAACGCTTGAGTT

$\ldots \ldots \ldots \ldots \ldots$

$\ldots \ldots \ldots \ldots \ldots$

$\ldots \ldots \ldots \ldots \ldots$

$\ldots \ldots \ldots \ldots \ldots$

$\ldots \ldots \ldots \ldots \ldots$

$\ldots \ldots \ldots \ldots \ldots$

$\ldots \ldots \ldots \ldots \ldots$

n.............

$\ldots \ldots \ldots \ldots$

AAGCCG

$\ldots \ldots$

$\ldots \ldots$

$\ldots \ldots$

$\cdots \cdots$

$\cdots \cdots$

$\cdots \cdots$

$\cdots \cdots$

$\cdots \cdots$

$\ldots \ldots$

CGCCGCGAAGCGGCG T CGGCTT G AACGAATTGT*TAGAC...

$\ldots \ldots \ldots \ldots \ldots$ A $\ldots \ldots . \ldots \ldots \ldots$

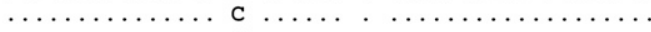

$\ldots \ldots \ldots \ldots \ldots, \mathrm{G} \ldots \ldots \ldots \ldots$

$\ldots \ldots \ldots \ldots \ldots \ldots \ldots \ldots$

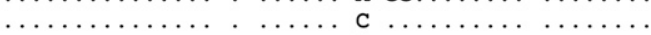

$\ldots \ldots \ldots \ldots \ldots$ т $\ldots \ldots \ldots \ldots \ldots$

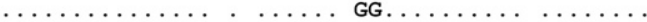

$\ldots$ A $\ldots \ldots$ C $\ldots \ldots \ldots \ldots \ldots \ldots$

......... G $\ldots \ldots$ T $\ldots \ldots \ldots \ldots$

$\mathbf{F}$

$\begin{array}{lr}\text { WT } & 5^{\prime}-\ldots \text { ATCTAACGCTTGAGTT AAGCCG } \\ \text { IBS ts } & \ldots \ldots \ldots \text { AA. } \ldots \ldots\end{array}$

CGCCGCGAAGCGGCG T CGGCTT G AACGAATTGT $*$ TAGAC...

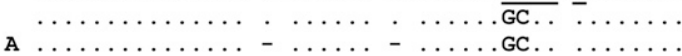

FIGURE 3. Sequence alignments of the att $C_{a a d A l}$ mutants tested as homing sites in this study. $(A)$ Mutation of the stem lengths; $(B)$ mutation of the stem pairings; $(C)$ mutation of IBS1 or IBS3; $(D)$ deletion of the extrahelical bases; $(E)$ mutation of the extrahelical bases; and $(F)$ inversion of the intron binding sites with or without the extrahelical bases. The figure shows only the bottom-strand sequences of the wild-type (WT) and mutant $a t t C_{a a d A 1}$ sites. Capital letters, attC sequences; lower case letters, pACYC184 sequences; gray shading and Vienna outputs, nucleotide pairings from a potential single strand stem-loop structure determined using MFOLD. Asterisk $\left({ }^{*}\right)$, intron homing site; underlined bases, IBS1 (TTGT) and IBS3 (T); dot, sequence identity to WT; dash, base deletion.

disruptions of the stem pairings strongly decrease the mobility frequency. To assess whether inhibition of mobility into stem mutants is a consequence of the absence of the extrahelical bases ( $\mathrm{T}$ and $\mathrm{G}$ ), we cloned three $a t t C$ mutants deleting either the T38 or G45 bulge bases or both (Figs. 1C, 3D). Deletion of the thymine at position 38 (T38) resulted in the inhibition of the homing reaction in att $C_{\text {aadAl }}$ in vivo (Fig. 5). The mobility frequency of S.ma.I2 into the $\Delta \mathrm{T}$ mutant was $>18$-fold lower than into the WT (LAG) site (Table 1). Nevertheless, Figure 4 shows that we were able to amplify homing events into the $\Delta \mathrm{T}$ mutant bottom strand, showing that the T38 bulge base is not essential for attC site recognition but enhances the mobility frequency. Sequencing of the PCR amplicon from the $5^{\prime}$ exon-intron integration junction within the $\Delta \mathrm{T}$ mutant (Fig. 4) confirmed the specificity of the intron for the homing site $5^{\prime}$-TTGT/T (data not shown). Deletion of the guanine at position 45 (G45) had no effect on target site recognition by the intron. The $\Delta \mathrm{G}$ mutant had mobility frequencies similar to those of the WT (LAG) site. Deletion of both T38 and G45 bases ( $\Delta \mathrm{T}+\Delta \mathrm{G}$ mutant) resulted in mobility frequencies comparable to $\Delta \mathrm{T}$ alone, and a weak PCR amplicon corresponding to specific bottom-strand insertion was observed (Fig. 4). 
TABLE 1. In vivo mobility frequencies of S.ma.I2 into WT and mutant attC $C_{a a d A 1}$ sites

\begin{tabular}{|c|c|c|c|}
\hline $\begin{array}{l}\text { Target } \\
\text { attC } C_{\text {aadA1 } 1}\end{array}$ & Orientation $^{\mathrm{a}}$ & $\begin{array}{l}\text { Homing } \\
\text { strand }^{b}\end{array}$ & $\begin{array}{c}\text { S.ma.I2 } \\
\text { mobility frequency } \\
(\%)^{\mathrm{c}}\end{array}$ \\
\hline WT & LAG & bs & $18.0 \pm 2.5$ \\
\hline WT & LEAD & bs & $<1.0$ \\
\hline$\Delta 1$ & LAG & bs & $8.0 \pm 0.7$ \\
\hline$\Delta 2$ & LAG & bs & $9.4 \pm 4.2$ \\
\hline$\Delta 3$ & LAG & bs & $10.1 \pm 3.8$ \\
\hline$\Delta 4$ & LAG & bs & $9.7 \pm 3.9$ \\
\hline$\Delta 5$ & LAG & bs & $1.8 \pm 1.0$ \\
\hline$\Delta 6$ & LAG & bs & $<1.0$ \\
\hline$\Delta 7$ & LAG & bs & $<1.0$ \\
\hline Stem mut. & LAG & bs & $<1.0$ \\
\hline IBS1-1 & LAG & bs & $<1.0$ \\
\hline IBS1-2 & LAG & bs & $<1.0$ \\
\hline IBS1-3 & LAG & bs & $<1.0$ \\
\hline IBS3 & LAG & bs & $4.6 \pm 2.9$ \\
\hline$\Delta \mathrm{T}$ & LAG & bs & $<1.0$ \\
\hline$\Delta \mathrm{G}$ & LAG & bs & $19.6 \pm 4.2$ \\
\hline$\Delta \mathrm{T}+\Delta \mathrm{G}$ & LAG & bs & $<1.0$ \\
\hline T38A & LAG & bs & $7.8 \pm 1.8$ \\
\hline T38C & LAG & bs & $15.2 \pm 3.6$ \\
\hline T38G & LAG & bs & $14.0 \pm 3.7$ \\
\hline G45A & LAG & bs & $12.3 \pm 4.9$ \\
\hline G45C & LAG & bs & $24.2 \pm 10.4$ \\
\hline G45T & LAG & bs & $13.3 \pm 2.9$ \\
\hline G45GG & LAG & bs & $<1.0$ \\
\hline$T 38 A+G 45 C$ & LAG & bs & $11.3 \pm 3.5$ \\
\hline $\mathrm{T} 38 \mathrm{G}+\mathrm{G} 45 \mathrm{~T}$ & LAG & bs & $15.0 \pm 6.5$ \\
\hline IBS ts & LAG & bs and ts & $<1.0$ \\
\hline IBS ts & LEAD & bs and ts & $<1.0$ \\
\hline IBS + bulges ts & LAG & ts & $<1.0$ \\
\hline IBS + bulges ts & LEAD & ts & $14.4 \pm 6.0$ \\
\hline
\end{tabular}

${ }^{a}$ Orientation of the attC bottom-strand sequence relative to the direction of DNA replication.

${ }^{\mathrm{b}}$ S.ma.I2 homing specificity (as detected by PCR on Fig. 4) for the attC bottom strand (bs) and/or top strand (ts).

${ }^{\mathrm{c}}$ The percentage of recipient plasmids that received the intron. Mobility frequencies were determined by colony patch hybridization with an intron-specific probe and are the mean of positive hybridization signals \pm standard deviation of at least three independent experiments in each case (see Materials and Methods).

\section{Mutation of the extrahelical bases}

Compilation of several integron cassette attC sites and folding of the bottom-strand sequence into the most probable stem-loop motifs showed that the two asymmetrical bases in the stem are a conserved thymine and guanine (Stokes et al. 1997; MacDonald et al. 2006). In order to determine if S.ma.I2 homing involves a specific bulge base, nine different mutants of the extrahelical bases T38 and G45 were examined (Fig. 3E). We observed that substitution of T38 with an adenine (T38A mutant) resulted in a more than twofold decrease of the intron mobility frequency compared with WT (LAG) (Fig. 5; Table 1). However, such differences were not observed with the T38C and T38G mutants. Substitution of G45 with any of the alternative bases (G45A, G45C, and G45T mutants) did not alter intron homing (Fig. 5). In the G45A mutant, additional mutations where made in order to prevent bulging of one of the two adjacent adenines (A46 or A47) instead of the G45A in the folded structure. In the G45GG mutant, another guanine was inserted next to the G45. This unusual mutant was tested as a homing site because it was previously shown that a GG bulge mutant of the att $C_{\text {aadA1 } 1}$ resulted in a complete loss of IntI1 integrase binding in vitro (Johansson et al. 2004). Strikingly, this mutant displayed $<1.0 \%$ homing efficiency, whereas the $\Delta \mathrm{G}$ mutant showed homing levels comparable to those of WT (LAG). Figure 4 shows that we successfully amplified homing events into the GG mutant bottom strand. Sequencing confirmed the specificity of the intron for the homing site $5^{\prime}$-TTGT/T (data not shown). Finally, intron mobility was tested using att $C_{a a d A 1}$ mutants for which extrahelical bases were either changed for the complementary base (T38A + G45C) or exchanged between themselves $(\mathrm{T} 38 \mathrm{G}+\mathrm{G} 45 \mathrm{~T})$. The T38A + G45C mutant showed a weak $(\sim 1.5$-fold $)$ decrease of homing frequency compared with WT (LAG) (most probably due to the T38A mutation), whereas no inhibition of the mobility was observed with the T38G + G45T mutant (Table 1).

\section{Inversion of the intron binding sites with or without the extrahelical bases}

Class IIC-attC introns identified so far are specifically inserted into attC bottom strands. Quantitative results show the unexpected importance of the $\mathrm{T}$ extrahelical base for a high level of mobility into $a t t C_{a a d A l}$ (above). A parallel can be drawn with integron integrase, for which the $\mathrm{T}$ base dictates the position of the integrase monomer along attC bottom strands (MacDonald et al. 2006). It was shown that minor alterations of the $a t t C_{a a d A l}$, involving nested inversions of both the bulge bases ( $T$ and $G$ ) and central loop, resulted in strong integrase binding to the top strand with the reciprocal loss of binding to the bottom strand (Johansson et al. 2004). Here, in order to change the S.ma.I2 bottom strand specificity, two minimal att $C_{a a d A 1}$ inversions were examined (Fig. 3F). First, intron-binding sequences, IBS1 and IBS3 (5'-TTGT/T), were symmetrically moved from the attC bottom strand to the top strand in the "IBS ts" (LAG) mutant. This inversion leads to an $>18$-fold decrease of mobility compared with WT (LAG) (Table 1). Interestingly, Figure 4 shows two weak PCR amplicons corresponding to mobility events that occurred in either strand. Similar homing frequencies $(<1.0 \%)$ and PCR results were obtained when the orientation of the target site was inverted relative to the direction of plasmid replication ("IBS ts" LEAD). Sequencing of the two PCR amplicons obtained from either LAG or LEAD mutant clones confirmed intron mobility into the new top strand IBS motifs, 5'-TTGT/T (IBS1/IBS3), but also into the 

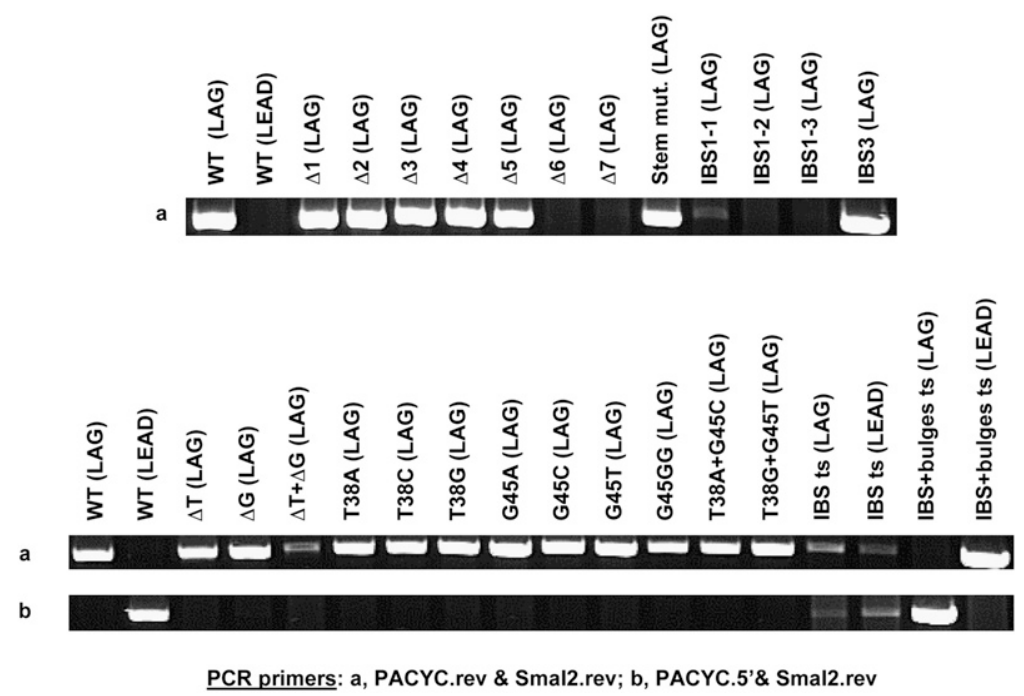

FIGURE 4. S.ma.I2 in vivo mobility results into att $C_{\text {aadAl }}$ mutants. Agarose gels of the PCR products using primer pair " $a$ " or " $\mathrm{b}$ " in order to detect the orientation of intron insertion. The figure shows the $5^{\prime}$ intron-exon integration junction (see Fig. 2 for primer position). "WT," "IBS ts," and "IBS + bulges ts" att $C_{a a d A 1}$ were cloned in both orientations (LAG or LEAD) relative to the direction of DNA replication in pACYC184 (see Materials and Methods).

bottom strand sequence, 5'-GCGT/T (IBS1/IBS3) (Fig. 6) corresponding to the mutated wild-type IBS motifs $\left(5^{\prime}\right.$-TTGT/T $\rightarrow$ 5'-GCGT/T). Second, intron-binding sequences and bulge bases (T38 and G45) were symmetrically moved from the attC bottom strand to the top strand within the "IBS+bulges ts" (LAG) mutant (Fig. 3F). These mutations led to an important inhibition of mobility into the attC site $(<1.0 \%$ homing efficiency), but strong PCR amplicons corresponding to specific top strand mobility events were observed (Fig. 4). Cloning of the same attC mutant into the opposite orientation ("IBS + bulges ts" LEAD), increased the intron mobility frequency to $14.4 \pm$ $6.0 \%$, comparable to WT (LAG) (Table 1). This increase of mobility is related to the same replication orientation bias observed with the WT attC site. Similarly, PCR amplification of the target site shows an increase of mobility events relative to the "IBS + bulges ts" (LAG) mutant (Fig. 5). Figure 4 shows a switch in the intron specificity for the attC top strand and the absence of mobility events into the bottom strand. Sequencing of the PCR amplicons confirmed the top-strand homing and specificity of the insertion site into the new IBS motifs (Fig. 6). Therefore, moving the IBS1-IBS3 sites to the opposite strand of the attC site retains high-level mobility only when accompanied by the bulge bases and the correct orientation with regard to DNA replication.

\section{DISCUSSION}

In this study, we searched for stem-loop features of att $C_{\text {aadA1 } 1}$ that could explain the site specificity of class
IIC-attC introns. Our results, based on qualitative PCR and quantitative hybridization methods, indicate that target specificity of S.ma.I2 occurs by recognition of short intron-binding sites $\left(5^{\prime}\right.$-TTGT/T as IBS1/IBS3, respectively) adjacent to a bulged hairpin structure within the att $C_{\text {aadAl }}$ bottom strand. This hairpin structure implies that the inverted-repeat motifs (1L-1R and $2 \mathrm{~L}-2 \mathrm{R})$, conserved among attC sites, could form a robust double-strand DNA-like structure in vivo, which can be bound by nonspecific phosphatebackbone interaction or specific base contacts by the intron RNP amino acids. It was shown for several group II introns, that in the absence of secondstrand cleavage, the intron RNA reverse splices at the DNA replication fork into either single-stranded or doublestranded DNA and then uses a nascent strand as primer for reverse transcription (Ichiyanagi et al. 2002; Zhong and Lambowitz 2003; Martinez-Abarca et al. 2004). We found that homing of S.ma.I2 displays a strong replication orientation bias and predominantly occurs using a nascent lagging DNA strand as a primer for reverse transcription of the inserted intron RNA. This bias reflects the absence of second-strand cleavage, because of the missing endonuclease domain of the IEP (Centron and Roy 2002), and suggests that S.ma.I2 reverse splices into single-stranded DNA targets at the DNA replication fork (Ichiyanagi et al. 2002; Zhong and Lambowitz 2003; Coros et al. 2005). Together, these results support the hypothesis that the DNA replication fork (rather than transcription bubbles) is the most probable source of single-strand DNA, allowing the attC bottom strand to fold into a stem-loop motif used by class IIC-attC introns as a structural determinant for target site recognition. In addition to group II introns, other mobile elements, including the IS605 group of insertion sequences and transposon $\mathrm{Tn} 7$, which use different mobility pathways, demonstrate lagging-strand preference during transposition (Peters and Craig 2001; Barabas et al. 2008).

The crystal structure of the VchIntIA- $\mathrm{VCR}_{\mathrm{bs}}$ complex confirms that the integrase binds the attC bottom strand as a DNA stem-loop with extrahelical bases (MacDonald et al. 2006). Our results suggest that S.ma.I2 uses the conserved attC structure in order to compensate for the shortened sequence-specific IBS1-EBS1 interaction and the missing EBS2-IBS2 motifs, which are normally involved in $5^{\prime}$ exon recognition. Therefore, there are two major determinants for att $C_{\text {aadAl }}$ recognition by S.ma.I2 in vivo. First, a fourbase IBS1 (TTGT) and a one-base IBS3 (T) base pair to 


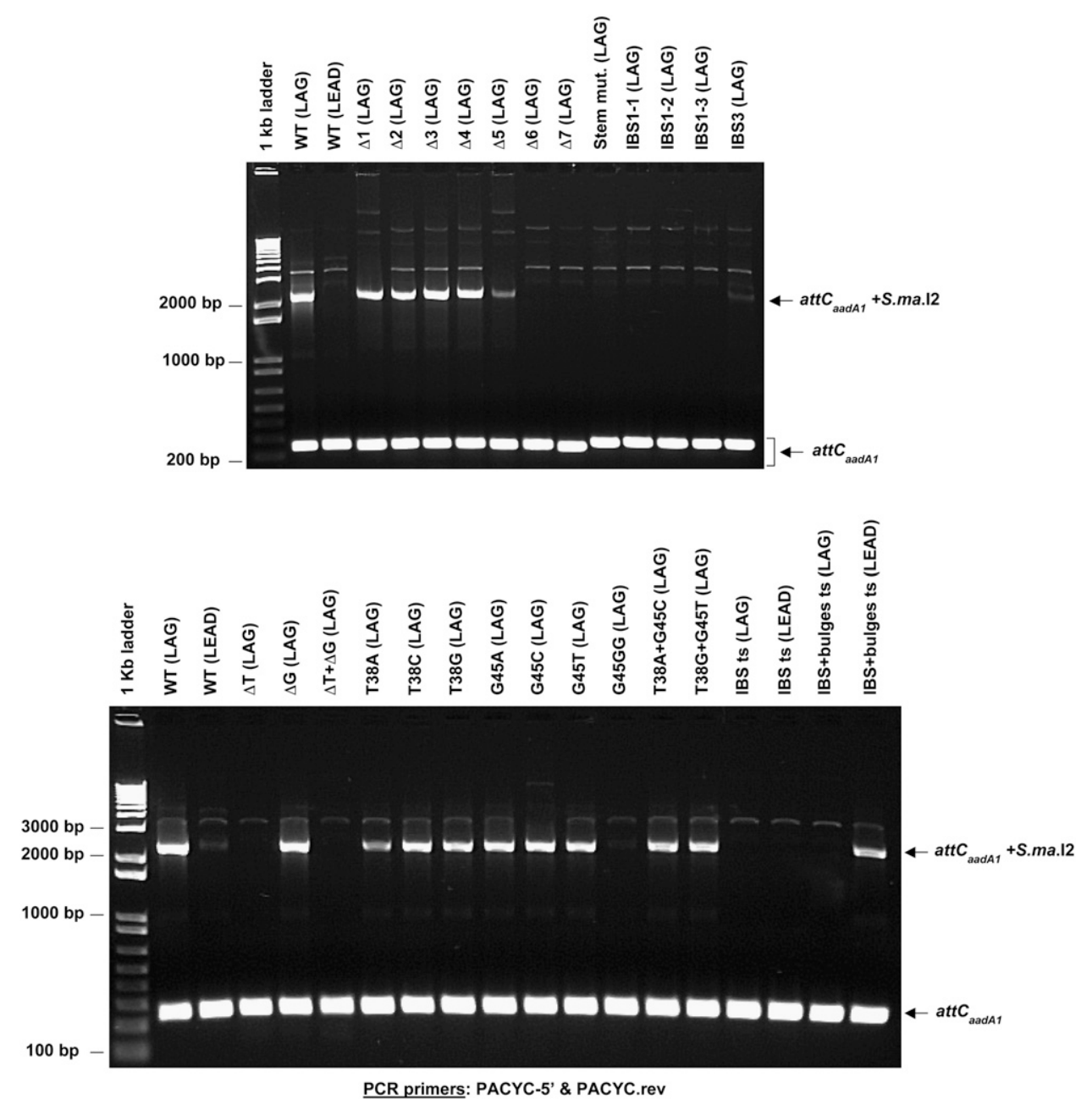

FIGURE 5. S.ma.I2 in vivo mobility results into $a t t C_{a a d A 1}$ mutants. Agarose gels of the PCR products using PACYC-5' and PACYC.rev primers in order to detect variations in the degree of intron insertion for each mutant compared with the WT site (see Fig. 2 for primer position). The Figure shows attCs that were and were not targeted by S.ma.I2.

complementary intron RNA exon-binding sites (as predicted by its secondary structure), and are conserved among several targeted attC sites (Quiroga et al. 2008). We showed that mutations of the IBS1 and (to a lesser degree) IBS3 motifs strongly inhibited the homing reaction into attCs in vivo. Second, there is an imperfect stem-loop motif adjacent to the intron binding sites. Compilation of insertion sites of several subgroup IIC introns shows that a stem-loop structure is located a few bases $(\leq 8)$ before the intron binding sites (Robart et al. 2007). Assays of homing into mutated att $C_{a a d A 1}$ sites showed mobility into a minimal $5^{\prime}$-exon sequence folded into a 12-base stem and a three-base loop motif. The IBS motifs and the minimal $5^{\prime}$-exon sequence requirement identified in this study is consistent with the consensus structure for several class $\mathrm{C}$ targets (Robart et al. 2007). We showed that deletion of the $1 \mathrm{R}$ region of attC $(\Delta 1)$ resulted in a $\sim 50 \%$ diminution of the homing reaction in vivo (Table 1), while deletion of the region between $1 \mathrm{R}$ and $2 \mathrm{R}(\Delta 2-\Delta 4)$ gave no further loss of mobility. Additional deletions including half $(\Delta 5)$ or the entire $2 \mathrm{R}$ site $(\Delta 6$ and $\Delta 7$ ) resulted in a $\geq 90 \%$ inhibition of mobility. Further studies showed that mutations disrupting the stem before the central loop led to an important inhibition of intron mobility (Table 1). Interestingly, an identical stem mutation on att $C_{a a d A 7}$, which differs by two nucleotides from att $C_{\text {aadAl }}$ (Johansson et al. 2004), leads to a 10 -fold decrease of the class 1 integrase recombination frequency (Bouvier et al. 2005). Together, these results show that correct folding of the attC bottom strand is essential for substrate recognition, and suggest that S.ma.I2 makes DNA-protein contacts with the same attC site determinants (1L-1R and $2 \mathrm{~L}-2 \mathrm{R}$ subsites) as integron integrase (MacDonald et al. 2006; Johansson et al. 2009).

Sequence variation between the $2 \mathrm{~L}$ and $2 \mathrm{R}$ repeats of several attC sites reveals a lack of absolute sequence requirement in the stem except for two conserved extrahelical bases ( $\mathrm{T}$ and $\mathrm{G}$ ) that are usually separated by a 
A

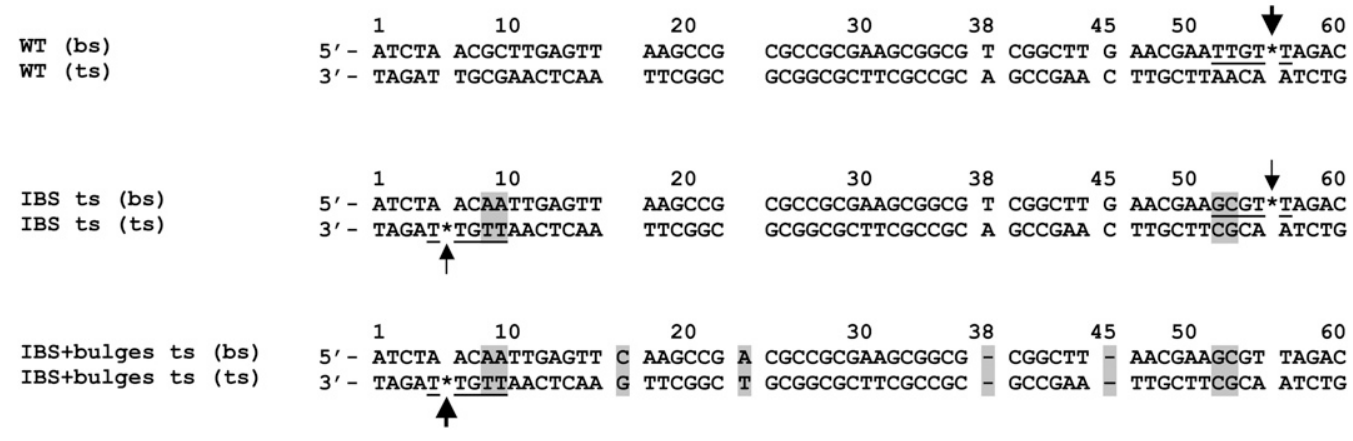

B

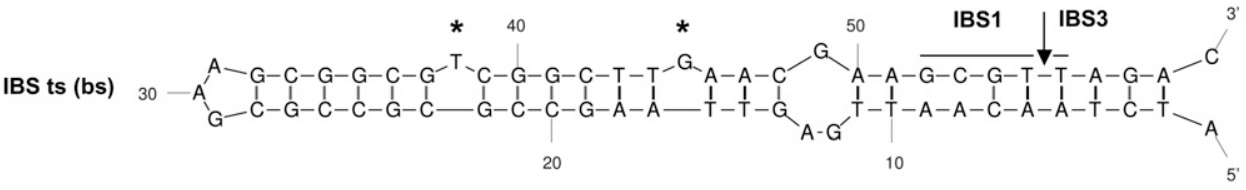

S ts (ts)

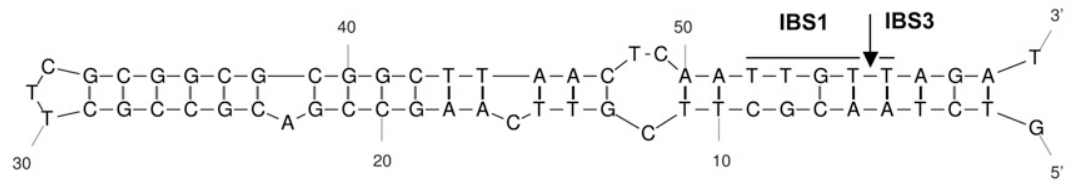

IBS+bulges ts (bs)
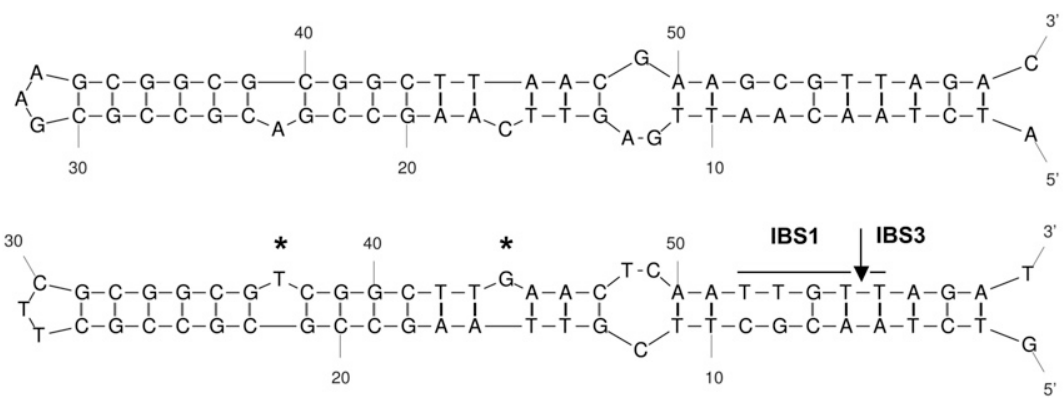

FIGURE 6. (A) Alignment of the sequenced PCR products from the S.ma.I2 mobility assays into the WT, "IBS ts," and "IBS + bulges ts" att $C_{\text {aadAl }}$ sites. Arrows indicate the intron homing site determined by PCR (Fig. 4). The thickness of the arrows corresponds to the mobility frequencies observed in Table 1. Asterisk $\left.{ }^{*}\right)$, intron homing site; underlined bases, intron binding site; highlighted bases, difference from the WT sequence; dash, base deletion. (B) Predicted stem-loop structures of the att $C_{a a d A 1}$ mutant top- and bottom-strand sequences based on MFOLD predictions. IBS1 and IBS3, intron binding sites 1 and 3; asterisk $\left(^{*}\right)$, extrahelical bases T38 and G45 upon folding of the attC bottom strand.

six-base spacer (Stokes et al. 1997). Mutation or deletion of the extrahelical base $\mathrm{T}$ results in inhibition of the homing reaction in vivo (Table 1). Interestingly, the crystal structure of the VchIntIA-VCR $\mathrm{VR}_{\mathrm{bs}}$ synapse showed that the extrahelical base $\mathrm{T}$ is bound by cis-interaction with one integrase subunit and is important for DNA site recognition (MacDonald et al. 2006). Deletion of the T or G extrahelical bases in $\mathrm{VCR}_{\mathrm{bs}}$ resulted in inhibition of excision frequency by VchIntIA integrase (MacDonald et al. 2006). Furthermore, it was demonstrated that a GG bulge mutant of the att $C_{a a d A 1}$ resulted in a complete loss of IntI1 integrase binding in vitro (Johansson et al. 2004). This mutation also inhibits S.ma.I2 homing into att $C_{a a d A 1}$, whereas deletion (or mutation for another base) of the G45 bulge has no effect on intron mobility. These results show that the G45 bulge is not recognized by S.ma.I2, and suggest that the G45GG mutation resulted in a change of the attC structure, which potentially inhibited an important contact with the T38 bulge. Consequently, these results suggest that $S . m a . I 2$ makes an unexpected additional DNA-protein contact with the T bulge, in order to position the intron RNP along the DNA, facilitate the IBS-EBS pairings, and enhance the homing frequency.

Similarly, recognition of extrahelical bases from stemloop motifs is also used by a particular class of DNA transposases coded by the IS605 group of insertion sequences. The transposases specifically bind to conserved 22-23 base imperfect inverted repeats located at the transposon ends. The crystal structure of the ISHp608 transposase from Helicobacter pylori with stem-loop DNA, from the folded 
inverted repeats, shows how DNA/protein interactions direct the transposase toward its cleavage site (Ronning et al. 2005). The transposase interacts with two unpaired thymine bases (separated by a six-base spacer) in these stem-loops, forming the basis for discrimination between the top and bottom strand stem-loops. Interactions between the ISHp608 transposase and the extrahelical bases are both protein side-chain and backbone interactions. Here, we demonstrated that full homing of the intron to the attC top strand was possible when both bulge bases and intron binding sites were symmetrically moved to the top strand (Fig. 6). Therefore, the conserved extrahelical bases (most importantly the $\mathrm{T}$ bulge) from the folded att $\mathrm{C}$ sites and the IBS1/IBS3 (5'-TTGT/T) motifs are the determinants for directing the intron to the attC bottom strand. Finally, these results suggest that class IIC-attC introns and integron integrases have convergently evolved to recognize attC sites and to tolerate $a t t C$ variation by recognition of a bulged hairpin DNA motif rather than a specific sequence.

\section{MATERIALS AND METHODS}

\section{Plasmids and media}

Plasmids are described in Supplemental Table 1. E. coli DH5- $\alpha$

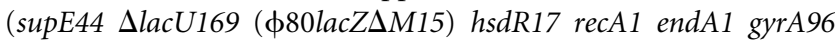
thi-1 relA1; Invitrogen) was grown in Luria-Bertani broth at $30^{\circ} \mathrm{C}$ or $37^{\circ} \mathrm{C}$ as indicated below. Antibiotics were used at the following concentrations: ampicillin (Ap), $100 \mu \mathrm{g} / \mathrm{mL}$; chloramphenicol $(\mathrm{Cm}), 68 \mu \mathrm{g} / \mathrm{mL}$. Isopropyl- $\beta$-D-thiogalactopyranoside (IPTG) was added to $1-\mathrm{mM}$ final concentration.

\section{Polymerase chain reaction procedures}

Polymerase chain reaction (PCR) for plasmid assembly and other PCR reactions used the Phusion DNA polymerase (Finnzymes) according to the manufacturer's instructions. PCR primers (Supplemental Table 2) were synthesized using the DNA Synthesizer ABI-3900 from Applied Biosystems, Inc.

\section{Nucleic acid folding}

Target site DNA single-stranded secondary structures were determined using the MFOLD 3.2 program (Zuker 2003).

\section{In vivo mobility assay}

Intron mobility was determined by PCR amplification of the targeted sites using an E. coli two-plasmid assay in which S.ma.I2 cloned into pUC18 inserts into mutant $a t t C_{a a d A 1}$ sites cloned into pACYC184 (Supplemental Table 1). pACYC184-based clones were obtained by cloning full-length complementary oligonucleotides (Supplemental Table 2) of each target site. Targets were cloned such that reverse transcription of the inserted intron RNA could potentially use either a nascent lagging (LAG) or leading (LEAD) DNA strand as a primer at the replication fork.

For each mobility assay, the intron donor $\left(\mathrm{Ap}^{\mathrm{R}}\right)$ and recipient $\left(\mathrm{Cm}^{\mathrm{R}}\right)$ plasmids were cotransformed into E. coli DH5- $\alpha$ compe- tent cells $\left(\operatorname{rec} A 1^{-}\right)$. One colony of each double transformant was grown in $5 \mathrm{~mL}$ of LB media in the presence of Ap and $\mathrm{Cm}$ at $30^{\circ} \mathrm{C}$ overnight. The preculture $(100 \mu \mathrm{L})$ was pelleted and inoculated in $5 \mathrm{~mL}$ of LB medium with both antibiotics at $37^{\circ} \mathrm{C}$ until O.D $\mathrm{D}_{600 \mathrm{~nm}}=$ 0.5. Expression of the intron was then induced by addition of $1 \mathrm{mM}$ IPTG, followed by incubation at $37^{\circ} \mathrm{C}$ for $3 \mathrm{~h}$. Plasmid DNAs were extracted using a QIAprep kit (Qiagen). Mobility was screened by PCR using different combinations of primers (Fig. 2; Supplemental Table 2) and insertion sites were confirmed by sequencing.

Homing frequency was determined by colony patch hybridization. Purified plasmids (50-100 ng) from S.ma.I2 mobility assays, were digested with five units of PvuI. This enzyme cuts only once in the intron donor plasmid, outside of the intron sequence. E. coli DH5- $\alpha$ cells were transformed with the digested products and plated on $\mathrm{LB} / \mathrm{Cm}$ plates to select for cells with recipient plasmids containing or lacking the intron. To calculate the intron mobility efficiency, measured by the percentage of recipient plasmids that received the intron, 100 isolated $\mathrm{Cm}^{\mathrm{R}}$ colonies were patched onto $\mathrm{LB} / \mathrm{Cm}$ plates. The patches were lifted onto nylon membranes and hybridized with $5^{\prime}$-end-labeled probe (SmaI2.for) using $\left[\gamma-{ }^{33} \mathrm{P}\right]$ ATP $(3000 \mathrm{Ci} / \mathrm{mmol}$; GE Healthcare). For each target site tested, positive colonies, randomly chosen, were cultured and the homing site-specificity confirmed by PCR amplification of the target site (using the PACYC-5' and PACYC.rev primers) and sequencing.

\section{SUPPLEMENTAL MATERIAL}

Supplemental material can be found at http://www.rnajournal.org.

\section{ACKNOWLEDGMENTS}

This work was supported by Canadian Institutes of Health Research (CIHR) Grant MT-13564 to P.H.R.

Received March 13, 2009; accepted May 4, 2009.

\section{REFERENCES}

Barabas O, Ronning DR, Guynet C, Hickman AB, Ton-Hoang B, Chandler M, Dyda F. 2008. Mechanism of IS200/IS605 family DNA transposases: Activation and transposon-directed target site selection. Cell 132: 208-220.

Bonen L, Vogel J. 2001. The ins and outs of group II introns. Trends Genet 17: 322-331.

Bouvier M, Demarre G, Mazel D. 2005. Integron cassette insertion: A recombination process involving a folded single strand substrate. EMBO J 24: 4356-4367.

Centron D, Roy PH. 2002. Presence of a group II intron in a multiresistant Serratia marcescens strain that harbors three integrons and a novel gene fusion. Antimicrob Agents Chemother 46: 1402-1409.

Coros CJ, Landthaler M, Piazza CL, Beauregard A, Esposito D, Perutka J, Lambowitz AM, Belfort M. 2005. Retrotransposition strategies of the Lactococcus lactis Ll.LtrB group II intron are dictated by host identity and cellular environment. Mol Microbiol 56: 509-524.

Cousineau B, Smith D, Lawrence-Cavanagh S, Mueller JE, Yang J, Mills D, Manias D, Dunny G, Lambowitz AM, Belfort M. 1998. Retrohoming of a bacterial group II intron: Mobility via complete reverse splicing, independent of homologous DNA recombination. Cell 94: 451-462. 
Ferat JL, Michel F. 1993. Group II self-splicing introns in bacteria. Nature 364: 358-361.

Francia MV, Avila P, de la Cruz F, Garcia Lobo JM. 1997. A hot spot in plasmid $\mathrm{F}$ for site-specific recombination mediated by $\mathrm{Tn} 21$ integron integrase. J Bacteriol 179: 4419-4425.

Francia MV, Zabala JC, de la Cruz F, Garcia Lobo JM. 1999. The IntI1 integron integrase preferentially binds single-stranded DNA of the attC site. J Bacteriol 181: 6844-6849.

Granlund M, Michel F, Norgren M. 2001. Mutually exclusive distribution of IS1548 and GBSil, an active group II intron identified in human isolates of group B streptococci. J Bacteriol 183: $2560-2569$.

Guo H, Zimmerly S, Perlman PS, Lambowitz AM. 1997. Group II intron endonucleases use both RNA and protein subunits for recognition of specific sequences in double-stranded DNA. EMBO J 16: 6835-6848.

Hall RM, Brookes DE, Stokes HW. 1991. Site-specific insertion of genes into integrons: Role of the 59-base element and determination of the recombination cross-over point. Mol Microbiol 5: 19411959.

Ichiyanagi K, Beauregard A, Lawrence S, Smith D, Cousineau B, Belfort M. 2002. Retrotransposition of the Ll.LtrB group II intron proceeds predominantly via reverse splicing into DNA targets. Mol Microbiol 46: 1259-1272.

Jacquier A, Michel F. 1987. Multiple exon-binding sites in class II selfsplicing introns. Cell 50: 17-29.

Jimenez-Zurdo JI, Garcia-Rodriguez FM, Barrientos-Duran A, Toro N. 2003. DNA target site requirements for homing in vivo of a bacterial group II intron encoding a protein lacking the DNA endonuclease domain. J Mol Biol 326: 413-423.

Johansson C, Kamali-Moghaddam M, Sundstrom L. 2004. Integron integrase binds to bulged hairpin DNA. Nucleic Acids Res 32: 4033-4043.

Johansson C, Boukharta L, Eriksson J, Aqvist J, Sundstrom L. 2009. Mutagenesis and homology modeling of the $\operatorname{Tn} 21$ integron integrase IntI1. Biochemistry 48: 1743-1753.

Lambowitz AM, Zimmerly S. 2004. Mobile group II introns. Annu Rev Genet 38: 1-35.

MacDonald D, Demarre G, Bouvier M, Mazel D, Gopaul DN. 2006. Structural basis for broad DNA-specificity in integron recombination. Nature 440: 1157-1162.

Martinez-Abarca F, Toro N. 2000. Group II introns in the bacterial world. Mol Microbiol 38: 917-926.

Martinez-Abarca F, Garcia-Rodriguez FM, Toro N. 2000. Homing of a bacterial group II intron with an intron-encoded protein lacking a recognizable endonuclease domain. Mol Microbiol 35: 1405-1412.

Martinez-Abarca F, Barrientos-Duran A, Fernandez-Lopez M, Toro N. 2004. The RmIntl group II intron has two different retrohoming pathways for mobility using predominantly the nascent lagging strand at DNA replication forks for priming. Nucleic Acids Res 32: 2880-2888.

Matsuura M, Saldanha R, Ma H, Wank H, Yang J, Mohr G, Cavanagh S, Dunny GM, Belfort M, Lambowitz AM. 1997. A bacterial group II intron encoding reverse transcriptase, maturase, and DNA endonuclease activities: Biochemical demonstration of maturase activity and insertion of new genetic information within the intron. Genes \& Dev 11: 2910-2924.

Mazel D. 2006. Integrons: Agents of bacterial evolution. Nat Rev Microbiol 4: 608-620.

Michel F, Ferat JL. 1995. Structure and activities of group II introns. Anпu Rev Biochem 64: 435-461.
Munoz-Adelantado E, San Filippo J, Martinez-Abarca F, GarciaRodriguez FM, Lambowitz AM, Toro N. 2003. Mobility of the Sinorhizobium meliloti group II intron RmInt1 occurs by reverse splicing into DNA, but requires an unknown reverse transcriptase priming mechanism. J Mol Biol 327: 931-943.

Peters JE, Craig NL. 2001. Tn7 recognizes transposition target structures associated with DNA replication using the DNAbinding protein TnsE. Genes \& Dev 15: 737-747.

Podar M, Chu VT, Pyle AM, Perlman PS. 1998. Group II intron splicing in vivo by first-step hydrolysis. Nature 391: 915-918.

Pyle AM, Lambowitz AM. 2006. Group II introns: Ribozymes that splice RNA and invade DNA. In The RNA World (eds. RF Gesteland et al.), pp 469-505. Cold Spring Harbor Laboratory Press, Cold Spring Harbor, NY.

Quiroga C, Roy PH, Centron D. 2008. The S.ma.I2 class C group II intron inserts at integron attC sites. Microbiology 154: 1341-1353.

Robart AR, Seo W, Zimmerly S. 2007. Insertion of group II intron retroelements after intrinsic transcriptional terminators. Proc Natl Acad Sci 104: 6620-6625.

Ronning DR, Guynet C, Ton-Hoang B, Perez ZN, Ghirlando R, Chandler M, Dyda F. 2005. Active site sharing and subterminal hairpin recognition in a new class of DNA transposases. Mol Cell 20: $143-154$.

Simon DM, Clarke NA, McNeil BA, Johnson I, Pantuso D, Dai L, Chai D, Zimmerly S. 2008. Group II introns in eubacteria and archaea: ORF-less introns and new varieties. RNA 14: 17041713.

Singh NN, Lambowitz AM. 2001. Interaction of a group II intron ribonucleoprotein endonuclease with its DNA target site investigated by DNA footprinting and modification interference. $J \mathrm{Mol}$ Biol 309: 361-386.

Stokes HW, Hall RM. 1989. A novel family of potentially mobile DNA elements encoding site-specific gene-integration functions: Integrons. Mol Microbiol 3: 1669-1683.

Stokes HW, O'Gorman DB, Recchia GD, Parsekhian M, Hall RM. 1997. Structure and function of 59-base element recombination sites associated with mobile gene cassettes. Mol Microbiol 26: 731745 .

Toor N, Hausner G, Zimmerly S. 2001. Coevolution of group II intron RNA structures with their intron-encoded reverse transcriptases. RNA 7: 1142-1152.

Toor N, Robart AR, Christianson J, Zimmerly S. 2006. Self-splicing of a group IIC intron: $5^{\prime}$ exon recognition and alternative $5^{\prime}$ splicing events implicate the stem-loop motif of a transcriptional terminator. Nucleic Acids Res 34: 6461-6471.

Toor N, Keating KS, Taylor SD, Pyle AM. 2008. Crystal structure of a self-spliced group II intron. Science 320: 77-82.

Toro N. 2003. Bacteria and Archaea Group II introns: Additional mobile genetic elements in the environment. Environ Microbiol 5: 143-151.

Zhong J, Lambowitz AM. 2003. Group II intron mobility using nascent strands at DNA replication forks to prime reverse transcription. EMBO J 22: 4555-4565.

Zimmerly S, Guo H, Eskes R, Yang J, Perlman PS, Lambowitz AM. 1995. A group II intron RNA is a catalytic component of a DNA endonuclease involved in intron mobility. Cell 83: 529-538.

Zimmerly S, Hausner G, Wu X. 2001. Phylogenetic relationships among group II intron ORFs. Nucleic Acids Res 29: 12381250.

Zuker M. 2003. Mfold web server for nucleic acid folding and hybridization prediction. Nucleic Acids Res 31: 3406-3415. 

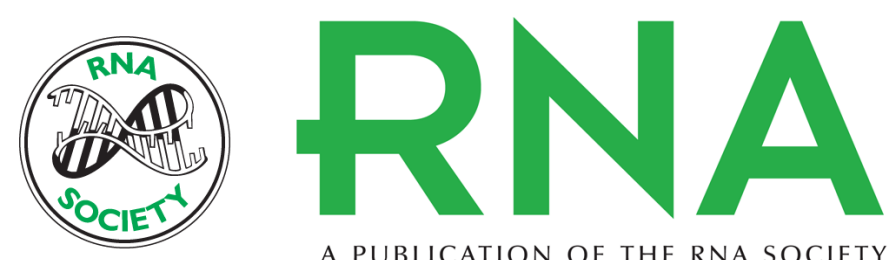

A PUBLICATION OF THE RNA SOCIETY

\title{
Group IIC intron mobility into attC sites involves a bulged DNA stem- loop motif
}

\author{
Grégory Léon and Paul H. Roy
}

RNA 2009 15: 1543-1553 originally published online June 9, 2009

Access the most recent version at doi:10.1261/rna.1649309

\section{Supplemental http://rnajournal.cshlp.org/content/suppl/2009/06/11/rna.1649309.DC1 \\ Material}

References This article cites 43 articles, 12 of which can be accessed free at: http://rnajournal.cshlp.org/content/15/8/1543.full.html\#ref-list-1

\section{License}

Email Alerting Receive free email alerts when new articles cite this article - sign up in the box at the Service top right corner of the article or click here.

To subscribe to RNA go to:

http://rnajournal.cshlp.org/subscriptions 